\title{
On the Maximum Displacement and Static Buckling of a Circular Cylindrical Shell
}

\author{
G. E. Ozoigbo ${ }^{*}$, A. M. Ette ${ }^{2}$ \\ ${ }^{1}$ Department of Mathematics/Computer Science/Statistics \& Informatics, Alex Ekwueme Federal University Ndufu-Alike, Ikwo, \\ Ebonyi State, Nigeria \\ ${ }^{2}$ Department of Mathematics, Federal University of Technology, Owerri, Imo State, Nigeria \\ Email: *geraldozoigbo@yahoo.co.uk, tonimonsette@yahoo.com
}

How to cite this paper: Ozoigbo, G.E. and Ette, A.M. (2019) On the Maximum Displacement and Static Buckling of a Circular Cylindrical Shell. Journal of Applied Mathematics and Physics, 7, 2868-2882. https://doi.org/10.4236/jamp.2019.711196

Received: August 18, 2019

Accepted: November 18, 2019

Published: November 21, 2019

Copyright ( 2019 by author(s) and Scientific Research Publishing Inc. This work is licensed under the Creative Commons Attribution International License (CC BY 4.0).

http://creativecommons.org/licenses/by/4.0/

\begin{abstract}
The static buckling load of an imperfect circular cylindrical shell is here determined asymptotically with the assumption that the normal displacement can be expanded in a double Fourier series. The buckling modes considered are the ones that are partly in the shape of imperfection, and partly in the shape of some higher buckling mode. Simply-supported boundary conditions are considered and the maximum displacement and the static buckling load are evaluated nontrivially. The results show, among other things, that generally the static buckling load, $\lambda_{s}$ decreases with increased imperfection and that the displacement in the shape of imperfection gives rise to the least static buckling load.
\end{abstract}

\section{Keywords}

Static, Maximum Displacement, Circular, Cylindrical Shell

\section{Introduction}

Cylindrical shells have wide engineering applications such as in the construction and study of aircraft, spacecraft and nuclear reactor, tanks for liquid and gas storage and pressure vessels, etc. The analyses of the buckling of cylindrical shells under various loading conditions have been made in the past years and both theoretical and experimental studies have been considered just as in [1] and [2]. Earlier studies on the buckling of shells were done by [3] [4] [5] [6] and [7], while Amazigo and Frazer [8], studied the buckling under external pressure of cylindrical shells with dimple-shaped initial imperfection. It would be recalled that Budiansky and Amazigo [9], investigated the buckling of infinitely long imperfect cylindrical shells subjected to static loads and Ette and Onwuchekwa [10], 
equally studied the static buckling of an externally pressurized finite circular cylindrical shell using asymptotic method. In this regard, mention must be made of Lockhart and Amazigo [11], who used perturbation method to investigate the dynamic buckling of finite circular cylindrical shells with small arbitrary geometric imperfections under external step-loading. In the same way, Bich et al. [12], by using analytical approach, investigated the nonlinear static and dynamic buckling behaviour of eccentrically shallow shells and circular cylindrical shells based on Donnell shell theory. Relevant studies on the buckling analysis were investigated in [13] [14] [15] and [16] and Ette [17] [18] [19] [20] and [21].

In this study, we consider a statically loaded imperfect finite circular cylindrical shell and aim at determining the maximum displacement and the static buckling load for the case where the displacement is partly in the shape of imperfection and partly in some other buckling mode. The analysis is purely on the use of asymptotic expansions and perturbation procedures.

This analysis is organised as follows. We shall first write down the governing equations as in Amazigo and Frazer [8] and Budiansky and Amazigo [9]. Using the techniques of regular perturbation and asymptotics, we shall analytically determine a uniformly valid expression of the displacement which is followed determining by the maximum displacement. Lastly, we shall reverse the series of maximum displacement and determine the static buckling load.

\section{Formulation}

As in [11], the general Karman-Donnell equation of motion and the compatibility equation governing the normal deflection $W(X, Y)$ and Airy stress function $F(X, Y)$ for cylindrical shell, of length $L$, radius $R$, thickness $h$, bending stiffness $D=\frac{E h^{3}}{12\left(1-v^{2}\right)}$ (where $E$ and $v$ are the Young's modulus and Poisson's ratio respectively), mass per unit area $\rho$, subjected to external pressure per unit area $P$, are

$$
\begin{gathered}
\frac{1}{E h} \nabla^{4} F-\frac{1}{R} W_{, X X}=-S\left(W, \frac{1}{2} W+\bar{W}\right) \\
D \nabla^{4} W+\frac{1}{R} F_{, X X}+P\left[\frac{1}{2} \alpha(W+\bar{W})_{, X X}+(W+\bar{W})_{, Y Y}\right]=S(W+\bar{W}, F) \\
W=W_{, X X}=F=F_{, X X}=0 \quad \text { at } X=0, L, \quad 0<X<\pi, \quad 0<Y<2 \pi .
\end{gathered}
$$

where, $X$ and $Y$ are the axial and circumferential coordinates respectively and $\bar{W}(X, Y)$, is a continuously differentiable stress-free and time independent imperfection. In this work, an alphabetic subscript placed after a comma indicates partial differentiation while $S$ is the symmetric bi-linear operator in $X$ and $Y$ given by

$$
S(P, Q)=P_{, X X} Q_{, Y Y}+P_{, Y Y} Q_{, X X}-2 P_{, X Y} Q_{, X Y}
$$

and $\nabla^{4}$, is the two-dimensional bi-harmonic operator defined by 


$$
\nabla^{4}=\left(\frac{\partial^{2}}{\partial X^{2}}+\frac{\partial^{2}}{\partial Y^{2}}\right)^{2}
$$

here, we shall neglect both axial and circumferential inertia and shall similarly assume simply-supported boundary conditions and neglect boundary layer effect by assuming that the pre-buckling deflection is constant.

As in [11] and [22], we now introduce the following non-dimensional quantities.

$$
\begin{gathered}
x=\frac{X \pi}{L}, \quad H=\frac{h}{R}, \quad \varepsilon \bar{w}=\frac{\bar{W}}{h}, \quad \lambda=\frac{L^{2} R P}{\pi^{2} D}, \quad \xi=\frac{L^{2}}{\pi^{2} R^{2}} \\
y=\frac{Y}{R}, \quad w=\frac{W}{h}, \quad K(\xi)=-\frac{A^{2}}{(1+\xi)^{2}}, \quad A=\frac{L^{2} \sqrt{12\left(1-v^{2}\right)}}{\pi^{2} R h}
\end{gathered}
$$

where, $v$ is Poisson's ratio and $\varepsilon$ is a small parameter which measures the amplitude of the imperfection while $L$ is the length of the cylindrical shell which is simply-supported at $x=0, \pi$.

We shall neglect boundary layer effect by assuming that the pre-buckling deflection is constant so that we let

$$
\begin{gathered}
F=-\frac{1}{2} P R\left(X^{2}+\frac{1}{2} \alpha Y^{2}\right)+\left(\frac{E h^{2} L^{2}}{\pi^{2} R(1+\xi)^{2}}\right) f \\
W=\frac{P R^{2}\left(1-\frac{1}{2} \alpha v\right)}{E h}+h w
\end{gathered}
$$

where, $P$ is the applied static load and $\lambda$ is the non-dimensional load amplitude. The first terms on the right hand sides of (2.7a) and (2.7b), are pre-buckling approximations, while the parameter $\alpha$, shall take the value $\alpha=1$, if pressure contributes to axial stress through the ends, otherwise $\alpha=0$, if pressure only acts laterally.

Substituting (2.7a) and (2.7b) into (2.1 and (2.2), using (2.5a) and (2.5b), and simplifying results to

$$
\bar{\nabla}^{4} f-(1+\xi)^{2} w_{, x x}=-H(1+\xi)^{2} s\left(w, \frac{1}{2} w+\varepsilon \bar{w}\right)
$$

and

$$
\begin{gathered}
\bar{\nabla}^{4} w-K(\xi) f_{, x x}+\lambda\left[\frac{1}{2} \alpha(w+\varepsilon \bar{w})_{, x x}+(w+\varepsilon \bar{w})_{, y y}\right]=-H K(\xi) s(w+\varepsilon \bar{w}, f) \\
w=w_{, x x}=f=f_{, x x}=0 \text { at } x=0, \pi, 0<x<\pi, 0<y<2 \pi, 0<\varepsilon \ll 1,(2
\end{gathered}
$$

where,

$$
s(P, Q)=P_{, x x} Q_{, y y}+P_{, y y} Q_{, x x}-2 P_{, x y} Q_{, x y}, \quad \bar{\nabla}^{4}=\left(\frac{\partial^{2}}{\partial x^{2}}+\xi \frac{\partial^{2}}{\partial y^{2}}\right)^{2}
$$

\section{Classical Buckling Load}

The classical buckling load $\lambda_{C}$ is the load that is required to buckle the asso- 
ciated linear perfect structure and its equations, from (2.8) and (2.9) are

$$
\bar{\nabla}^{4} f-(1+\xi)^{2} w_{, x x}=0
$$

and

$$
\begin{gathered}
\bar{\nabla}^{4} w-K(\xi) f_{, x x}+\lambda\left[\frac{1}{2} \alpha w_{, x x}+\bar{w}_{, y y}\right]=0 \\
w=w_{, x x}=0, \quad f=f_{, x x}=0 \quad \text { at } x=0, \pi .
\end{gathered}
$$

The solution to (3.1)-(3.3) is a superposition of the form

$$
(w, f)=\left(a_{m k}, b_{m k}\right) \sin \left(k y+\phi_{m k}\right) \sin m x
$$

where, $\left(a_{m k}, b_{m k}\right) \neq(0,0)$ and $\phi_{m k}$ is an inconsequential phase.

On substituting (3.4) into (3.1), using (3.3) and after lengthy simplification, we get

$$
b_{m k}=-\frac{(1+\xi)^{2} m^{2} a_{m k}}{\left(m^{2}+\xi k^{2}\right)^{2}}
$$

Substituting (3.5) into (3.2) and simplifying, yields

$$
\lambda=\frac{\left(m^{2}+\xi k^{2}\right)^{2}-\frac{K(\xi) m^{4}(1+\xi)^{2}}{\left(m^{2}+\xi k^{2}\right)^{2}}}{\frac{1}{2} \alpha m^{2}+\frac{k^{2} m^{4}(1+\xi)^{2} \xi}{\left(m^{2}+\xi k^{2}\right)^{2}}}
$$

Thus, if $n$ is the critical value of $k$ that minimizes $\lambda$, then, the value of $\lambda$ at $k=n$ was taken as the classical buckling load $\lambda_{C}$. Thus, in this case, we get

$$
\frac{\mathrm{d} \lambda}{\mathrm{d} k}=0
$$

Therefore, corresponding to $k=n$, we see that (3.6) is now equivalent to

$$
\lambda=\frac{\left(m^{2}+\xi n^{2}\right)^{2}-\frac{K(\xi) m^{4}(1+\xi)^{2}}{\left(m^{2}+\xi n^{2}\right)^{2}}}{\frac{1}{2} \alpha m^{2}+\frac{n^{2} m^{4}(1+\xi)^{2} \xi}{\left(m^{2}+\xi n^{2}\right)^{2}}}
$$

Usually, $m$ and $n$ take the values $m=1,2,3, \cdots$ and $n=0,1,2, \cdots$

We recall that [23] had assumed that $k$ varies continuously, and so, minimized $\lambda$ with respect to $k$. If $m=1$ is the nontrivial values of $m$ and we let $\zeta=\xi n^{2}$, then, we have

$$
\lambda_{C}=\frac{(1+\zeta)^{2}+\frac{A^{2}}{(1+\zeta)^{2}}}{\frac{1}{2} \alpha+\zeta}
$$

The corresponding displacement and Airy Stress function are 


$$
(w, f)=\left[\left\{1,-\left(\frac{1+\xi}{1+\zeta}\right)^{2}\right\}\right] a_{1 n} \sin \left(k y+\phi_{1 n}\right) \sin x
$$

\section{Static Theory}

In this section, we shall derive the equations satisfied by the displacement and Airy stress functions when the static load is applied.

Similar to (2.8) and (2.9), the structure satisfies the following equations at static loading

$$
\bar{\nabla}^{4} f-(1+\xi)^{2} w_{, x x}=-H(1+\xi)^{2} s\left(w, \frac{1}{2} w+\varepsilon \bar{w}\right)
$$

and

$$
\begin{gathered}
\bar{\nabla}^{4} w-K(\xi) f_{, x x}+\lambda\left[\frac{1}{2} \alpha(w+\varepsilon \bar{w})_{, x x}+\xi(w+\varepsilon \bar{w})_{, y y}\right]=-H K(\xi) s(w+\varepsilon \bar{w}, f) \\
w=w_{, x x}=f=f_{, x x}=0 \quad \text { at } x=0, \pi
\end{gathered}
$$

We now assume the following asymptotic expansions

$$
\left(\begin{array}{c}
w \\
f
\end{array}\right)=\sum_{i=1}^{\infty}\left(\begin{array}{c}
w^{(i)} \\
f^{(i)}
\end{array}\right) \varepsilon^{i}
$$

Substituting (4.4) into (4.1) and (4.2), and equating the coefficients of orders of $\varepsilon^{i}, i=1,2,3, \cdots$, the following equations are obtained

$$
\begin{aligned}
& \mathcal{O}(\varepsilon):\left\{\begin{array}{l}
\bar{\nabla}^{4} f^{(1)}-(1+\xi)^{2} w_{, x x}^{(1)}=0 . \\
\bar{\nabla}^{4} w^{(1)}-K(\xi) f_{, x x}^{(1)}+\lambda\left[\frac{1}{2} \alpha\left(w^{(1)}+\bar{w}\right)_{, x x}+\xi\left(w^{(1)}+\bar{w}\right)_{, y y}\right]=0
\end{array}\right. \\
& \mathcal{O}\left(\varepsilon^{2}\right):\left\{\begin{array}{l}
\bar{\nabla}^{4} f^{(2)}-(1+\xi)^{2} w_{, x x}^{(2)}=-H(1+\xi)^{2}\left[\frac{1}{2} s\left(w^{(1)}, w^{(1)}\right)+s\left(w^{(1)}, \bar{w}\right)\right] \\
\bar{\nabla}^{4} w^{(2)}-K(\xi) f_{, x x}^{(2)}+\lambda\left[\frac{1}{2} \alpha w_{, x x}^{(2)}+\xi w_{, y y}^{(2)}\right] \\
=-H K(\xi)\left[s\left(w^{(1)}, f^{(1)}\right)+s\left(\bar{w}, w^{(1)}\right)\right]
\end{array}\right. \\
& \mathcal{O}\left(\varepsilon^{3}\right):\left\{\begin{array}{l}
\bar{\nabla}^{4} f^{(3)}-(1+\xi)^{2} w_{, x x}^{(3)}=-H(1+\xi)^{2}\left[s\left(w^{(1)}, w^{(2)}\right)+s\left(w^{(2)}, \bar{w}\right)\right] \\
\bar{\nabla}^{4} w^{(3)}-K(\xi) f_{, x x}^{(3)}+\lambda\left[\frac{1}{2} \alpha w_{, x x}^{(3)}+\xi w_{, y y}^{(3)}\right] \\
=-H K(\xi)\left[s\left(w^{(1)}, f^{(2)}\right)+s\left(w^{(2)}, f^{(1)}\right)+s\left(\bar{w}, f^{(2)}\right)\right]
\end{array}\right.
\end{aligned}
$$

etc.

We seek solutions to (4.5)-(4.7) in the form

$$
\left(\begin{array}{c}
f^{(i)} \\
w^{(i)}
\end{array}\right)=\sum_{(k=1),(p=0)}^{\infty}\left[\left(\begin{array}{c}
f_{1}^{(i)} \\
w_{1}^{(i)}
\end{array}\right) \cos p y+\left(\begin{array}{c}
f_{2}^{(i)} \\
w_{2}^{(i)}
\end{array}\right) \sin p y\right] \sin k x
$$


and now assume

$$
\bar{w}(x, y)=\bar{a} \sin m x \sin n y
$$

As earlier obtained, we shall need the following simplifications

$$
\bar{\nabla}^{4}=\left(\frac{\partial^{2}}{\partial x^{2}}+\xi \frac{\partial^{2}}{\partial y^{2}}\right)^{2}=\left(\frac{\partial^{4}}{\partial x^{4}}+2 \xi \frac{\partial^{4}}{\partial x^{2} \partial y^{2}}+\xi^{2} \frac{\partial^{4}}{\partial y^{4}}\right)
$$

so that, if

$$
f^{(1)}=f_{\Gamma_{1}}^{(1)} \cos p y \sin k x
$$

then, we have

$$
\bar{\nabla}^{4} f^{(1)}=\left(k^{2}+\xi p^{2}\right)^{2} f_{\Gamma_{1}}^{(1)} \cos p y \sin k x, \quad \Gamma_{1}=1,2
$$

and, if

$$
f^{(2)}=f_{\Gamma_{2}}^{(2)} \sin p y \sin k x
$$

we get

$$
\bar{\nabla}^{4} f^{(2)}=\left(k^{2}+\xi p^{2}\right)^{2} f_{\Gamma_{2}}^{(2)} \sin p y \sin k x, \quad \Gamma_{2}=1,2
$$

We shall use the fact that

$$
K(\xi)=-\frac{A^{2}}{(1+\xi)^{2}}
$$

\section{Solution of Equations of First Order Perturbation}

The equations necessary here, from (4.5), are

$$
\bar{\nabla}^{4} f^{(1)}-(1+\xi)^{2} w_{, x x}^{(1)}=0
$$

and

$$
\bar{\nabla}^{4} w^{(1)}-K(\xi) f_{, x x}^{(1)}+\lambda\left[\frac{1}{2} \alpha\left(w^{(1)}+\bar{w}\right)_{, x x}+\xi\left(w^{(1)}+\bar{w}\right)_{, y y}\right]=0
$$

Substituting (4.8) and (4.9) into (4.11), using (4.10a), (4.10b) and (4.10c), multiplying the resultant equation through by $\cos n y \sin m x$ and integrating with respect to $y$ from 0 to $2 \pi$ and with respect to $x$ from 0 to $\pi$, we note that for $p=n, k=m$, we easily get

$$
f_{1}^{(1)}=-\frac{m^{2}(1+\xi)^{2} w_{1}^{(1)}}{\left(m^{2}+n^{2} \xi\right)^{2}}
$$

Similarly, by multiplying the resultant equation through by $\sin n y \sin m x$, and integrating with respect to $y$ from 0 to $2 \pi$ and with respect to $x$ from 0 to $\pi$, and for $p=n, k=m$, we obtain

$$
f_{2}^{(1)}=-\frac{m^{2}(1+\xi)^{2} w_{2}^{(1)}}{\left(m^{2}+n^{2} \xi\right)^{2}}
$$

In the same manner, substituting (4.8) and (4.9) into (4.12), assuming (4.10a), 
(4.10b) and (4.10c), thereafter multiplying the resultant equation by $\sin n y \sin m x$ and integrating with respect to $x$ from 0 to $\pi$ and $y$ from 0 to $2 \pi$, and for $p=n, k=m$, we get

$$
\left[\left(m^{2}+n^{2} \xi\right)^{2}-\lambda\left(\frac{1}{2} \alpha m^{2}+n^{2} \xi\right)\right] w_{2}^{(1)}+K(\xi) m^{2} V_{2}^{(1)}=\lambda \bar{a}\left(\frac{1}{2} \alpha m^{2}+n^{2} \xi\right)(4
$$

On substituting for $f_{2}^{(1)}$ from (4.14) and $K(\xi)$ from (4.10d) in (4.15) and simplifying, yields

$$
w_{2}^{(1)}=B_{0}
$$

where,

$$
\begin{aligned}
B_{0} & =\frac{\lambda \bar{a}\left(\frac{1}{2} \alpha m^{2}+n^{2} \xi\right)}{\varphi_{0}^{2}}, \\
\varphi_{0}^{2} & =\left(m^{2}+n^{2} \xi\right)^{2}+\left(\frac{m^{2} A}{m^{2}+n^{2} \xi}\right)^{2}-\lambda\left(\frac{1}{2} \alpha m^{2}+n^{2} \xi\right)
\end{aligned}
$$

Next, multiplying the resultant equation by $\cos n y \cos m x$ and integrating with respect to $x$ and $y$ from 0 to $\pi$ and 0 to $2 \pi$, respectively for $p=n, k=m$, and simplifying, we get

$$
w_{1}^{(1)}=0
$$

We therefore expect from (4.8) that for $i=1$

$$
w^{(1)}=B_{0} \sin m x \sin n y ; \quad f^{(1)}=-\Phi_{0} B_{0} \sin m x \sin n y, \quad \Phi_{0}=\frac{m^{2}(1+\xi)^{2}}{\left(m^{2}+n^{2} \xi\right)^{2}}(4
$$

\section{Solution of Equations of Second Order Perturbation}

Equations of the second order to be solved are from (4.6), namely

$$
\bar{\nabla}^{4} f^{(2)}-(1+\xi)^{2} w_{, x x}^{(2)}=-H(1+\xi)^{2}\left[\frac{1}{2} s\left(w^{(1)}, w^{(1)}\right)+s\left(w^{(1)}, \bar{w}\right)\right]
$$

and

$$
\begin{aligned}
& \bar{\nabla}^{4} w^{(2)}-K(\xi) f_{, x x}^{(2)}+\lambda\left[\frac{1}{2} \alpha w_{, x x}^{(2)}+\xi w_{, y y}^{(2)}\right] \\
& =-H K(\xi)\left[s\left(w^{(1)}, w^{(1)}\right)+s\left(\bar{w}, w^{(1)}\right)\right]
\end{aligned}
$$

Evaluating the symmetric bi-linear functions on the right hand sides of (4.20) and (4.21), substituting the same and simplifying, we get (after simplifying trigonometric terms)

$\bar{\nabla}^{4} f^{(2)}-(1+\xi)^{2} w_{, x x}^{(2)}=-H(1+\xi)^{2}(m n)^{2}\left[\left(\frac{1}{2} B_{0}^{2}+B_{0} \bar{a}\right)(\cos 2 m x+\cos 2 n y)\right]($

and

$$
\begin{aligned}
& \bar{\nabla}^{4} w^{(2)}-K(\xi) f_{, x x}^{(2)}+\lambda\left[\frac{1}{2} \alpha w_{, x x}^{(2)}+\xi w_{, y y}^{(2)}\right] \\
& =-H K(\xi)(m n)^{2}\left[\Phi_{0} B_{0}^{2}+\bar{a} B_{0}\right](\cos 2 m x+\cos 2 n y)
\end{aligned}
$$


Next we substitute (4.8) and (4.9) into (4.22), assuming (4.10a), (4.10b) and (4.10c), for $i=2$. Thereafter, we multiply the resultant equation through by $\cos 2 n y \sin m x$ and integrate with respect to $x$ and $y$, to get

$$
\begin{aligned}
& f_{1}^{(2)}=\Phi_{1}\left(\frac{1}{2} B_{0}^{2}+B_{0} \bar{a}\right)-\Phi_{2} w_{1}^{(2)}, \\
& \Phi_{1}=\frac{4 H m n^{2}(1+\xi)^{2}}{\pi\left(m^{2}+4 n^{2} \xi\right)^{2}}, \quad \Phi_{2}=\frac{m^{2}(1+\xi)^{2}}{\pi\left(m^{2}+4 n^{2} \xi\right)^{2}}, \quad m=\text { odd }
\end{aligned}
$$

Similarly, we next multiply the resultant equation by $\sin n y \sin m x$ and integrate as usual, and for $p=n, k=m$, we get

$$
f_{2}^{(2)}=-\frac{m^{2}(1+\xi)^{2} w_{2}^{(2)}}{\left(m^{2}+n^{2} \xi\right)^{2}} .
$$

Next, we substitute (4.8) and (4.9) into (4.23), using (4.10a), (4.10b) and (4.10c), for $i=2$, and then multiply the resultant equation through by $\cos 2 n y \sin m x$ and integrate, as usual for $p=2 n, k=m$ to get

$$
\begin{gathered}
w_{1}^{(2)}=\Phi_{5}, \quad \Phi_{5}=\Phi_{3}\left(B_{0}^{2} \Phi_{0}+B_{0} \Phi_{0} \bar{a}\right)+\Phi_{4}\left(\frac{1}{2} B_{0}^{2}+B_{0} \bar{a}\right) \\
\Phi_{3}=\frac{(m n)^{2} H K(\xi)}{\left(m^{2}+4 n^{2} \xi\right)^{2}+\left(\frac{m^{2} A}{m^{2}+4 n^{2} \xi}\right)^{2}-\lambda\left(\frac{1}{2} \alpha m^{2}+4 n^{2} \xi\right)} \\
\Phi_{4}=\frac{4 m^{3} n^{2} A^{2}}{\left(m^{2}+4 n^{2} \xi\right)^{2}\left[\left(m^{2}+4 n^{2} \xi\right)^{2}+\left(\frac{m^{2} A}{m^{2}+4 n^{2} \xi}\right)^{2}-\lambda\left(\frac{1}{2} \alpha m^{2}+4 n^{2} \xi\right)\right]}
\end{gathered}
$$

In the same manner, multiply the resultant equation by $\sin n y \sin m x$ and integrate with respect to $x$ and $y$, for $p=n, k=m$, and simplify to get

$$
w_{2}^{(2)}=0, \quad f_{2}^{(2)}=0
$$

Therefore, we observe from (4.8), and for $i=2$, that

$$
w^{(2)}=w_{1}^{(2)} \cos 2 n y \sin m x ; \quad f^{(2)}=f_{1}^{(2)} \cos 2 n y \sin m x,
$$

On substitution in (4.29) using (4.24) and in the first part of (4.26), we get

$$
\begin{aligned}
& w^{(2)}=\Phi_{5} \cos 2 n y \sin m x ; \quad f^{(2)}=\Phi_{10} \cos 2 n y \sin m x, \\
& \Phi_{10}=\left[\Phi_{1}\left(\frac{1}{2} B_{0}^{2}+B_{0} \bar{a}\right)-\Phi_{2} \Phi_{5}\right]
\end{aligned}
$$

\section{Solution of Equations of Third Order Perturbation}

The actual equations of the third order are from (4.7), namely

$$
\bar{\nabla}^{4} f^{(3)}-(1+\xi)^{2} w_{, x x}^{(3)}=-H(1+\xi)^{2}\left[s\left(w^{(1)}, w^{(2)}\right)+s\left(w^{(2)}, \bar{w}\right)\right]
$$

and 


$$
\begin{aligned}
& \bar{\nabla}^{4} w^{(3)}-K(\xi) f_{, x x}^{(3)}+\lambda\left[\frac{1}{2} \alpha w_{, x x}^{(3)}+\xi w_{, y y}^{(3)}\right] \\
& =-H K(\xi)\left[s\left(w^{(1)}, f^{(2)}\right)+s\left(w^{(2)}, f^{(1)}\right)+s\left(\bar{w}, f^{(2)}\right)\right]
\end{aligned}
$$

Evaluating the symmetric bi-linear functions on the right sides of (4.31) and (4.32) and substituting the same and simplifying, yields

$$
\begin{aligned}
& \bar{\nabla}^{4} f^{(3)}-(1+\xi)^{2} w_{, x x}^{(3)} \\
&=-\frac{1}{4} H(1+\xi)^{2}(m n)^{2}\left(\Phi_{5} B_{0}+\Phi_{5} \bar{a}\right)[9 \sin 3 n y-\sin n y \\
&-\cos 2 m x \sin 3 n y+9 \cos 2 m x \sin n y]
\end{aligned}
$$

and

$$
\begin{aligned}
\bar{\nabla}^{4} w^{(3)}-K(\xi) f_{, x x}^{(3)}+\lambda\left[\frac{1}{2} \alpha w_{, x x}^{(3)}+\xi w_{, y y}^{(3)}\right] \\
=-\frac{1}{4} H K(\xi)(m n)^{2}\left[-\Phi_{0} \Phi_{5} B_{0}+B_{0}\left\{\Phi_{1}\left(\frac{1}{2} B_{0}^{2}+B_{0} \bar{a}\right)-\Phi_{2} \Phi_{5}\right\}\right. \\
\left.+\bar{a}\left\{\Phi_{1}\left(\frac{1}{2} B_{0}^{2}+B_{0} \bar{a}\right)-\Phi_{2} \Phi_{5}\right\}\right][9 \sin 3 n y-\sin n y \\
-\cos 2 m x \sin 3 n y+9 \cos 2 m x \sin n y]
\end{aligned}
$$

We observe from the simplifications on the right hand sides of (4.33) and (4.34) that there will be four buckling modes generated $w_{i(r, p)}^{(3)}$ with their respective Airy stress functions $f_{i(r, p)}^{(3)}$. These buckling modes correspond to the following terms on the right hand sides of (4.33) and (4.34): $\sin 3 n y \sin m x$, $\sin n y \sin m x, \cos 2 m x \sin 3 n y$ and $\cos 2 m x \sin n y$.

However, of the four modes, it is only the mode in the shape of $\sin n y \sin m x$ that is in the shape of the imperfection. We shall consider this mode and the additional mode in the shape of $\sin 3 n y \sin m x$.

We now substitute (4.8) and (4.9) into (4.33), using (4.10a), (4.10b) and (4.10c), for $i=3$, and thereafter, multiply the resultant equation through by $\sin 3 n y \sin m x$ and integrate and for $k=m, p=3 n$, to get

$$
f_{2(m, 3 n)}^{(3)}=\frac{1}{\left(m^{2}+9 n^{2} \xi\right)^{2}}\left\{\frac{9}{\pi} H(1+\xi)^{2} m n^{2} A_{0}-m^{2}(1+\xi)^{2} w_{2(m, 3 n)}^{(3)}\right\}
$$

In the same way, we multiply the resultant equation by $\sin m x \sin n y$, integrate and for $k=m, p=n$, to get

$$
\begin{aligned}
& f_{2(m, n)}^{(3)}=\frac{1}{\left(m^{2}+n^{2} \xi\right)^{2}}\left\{\frac{1}{\pi} H(1+\xi)^{2} m n^{2} A_{0}-m^{2}(1+\xi)^{2} w_{2(m, n)}^{(3)}\right\}, \\
& A_{0}=B_{0}^{3} l_{0}, \quad l_{0}=\Phi_{5}\left(\frac{1}{B_{0}^{2}}+\frac{1}{B_{0}^{3}} \bar{a}\right)
\end{aligned}
$$

We next substitute (4.8) and (4.9) into (4.34), using (4.10a), (4.10b) and (4.10c), for $i=3$. Thereafter, we multiply the resultant equation by $\sin 3 n y \sin m x$, integrate and note that for, $k=m, p=3 n$, we get 


$$
\begin{aligned}
& w_{2(m, 3 n)}^{(3)}=\frac{\Theta_{0} A_{0}-\Theta_{1} A_{01}}{\left(m^{2}+9 n^{2} \xi\right)^{2}+\left(\frac{m^{2} A}{\left(m^{2}+9 n^{2} \xi\right)}\right)^{2}-\lambda\left(\frac{1}{2} \alpha m^{2}+9 n^{2} \xi\right)}, \\
& \Theta_{0}=\frac{9}{\pi\left(m^{2}+9 n^{2} \xi\right)^{2}} H m^{3} n^{2} A^{2}, \quad \Theta_{1}=\frac{9}{\pi} H K(\xi) m n^{2}, \\
& A_{01}=B_{0}^{3} l_{01}, \quad l_{01}=\frac{1}{2} \Phi_{1}-\frac{1}{B_{0}^{2}} \Phi_{0} \Phi_{5}+\frac{1}{B_{0}} \bar{a} \Phi_{1}-\frac{1}{B_{0}^{2}} \Phi_{2} \Phi_{5}
\end{aligned}
$$

Similarly, we multiply through by $\sin n y \sin m x$ and note that for $k=m, p=k$, we get

$$
\begin{aligned}
& w_{2(m, n)}^{(3)}=\frac{\Theta_{2} A_{01}-\Theta_{3} A_{0}}{\left(m^{2}+n^{2} \xi\right)^{2}+\left(\frac{m^{2} A}{\left(m^{2}+n^{2} \xi\right)}\right)^{2}-\lambda\left(\frac{1}{2} \alpha m^{2}+n^{2} \xi\right)}, \\
& \Theta_{3}=\frac{1}{\pi\left(m^{2}+n^{2} \xi\right)^{2}} H m^{3} n^{2} A^{2}, \quad \Theta_{2}=\frac{1}{\pi} H K(\xi) m n^{2}
\end{aligned}
$$

Thus, of the four non-zero buckling modes of this order and their respective Airy stress function, the ones we shall consider are

$$
\begin{aligned}
& w_{2(m, 3 n)}^{(3)} \sin 3 n y \sin m x, \quad w_{2(m, n)}^{(3)} \sin n y \sin m x, \\
& f_{2(m, 3 n)}^{(3)} \sin 3 n y \sin m x \text { and } f_{2(m, n)}^{(3)} \sin n y \sin m x
\end{aligned}
$$

As a summary so far, we can write the displacement and its respective Airy stress functions as

$$
\begin{aligned}
\left(\begin{array}{c}
w \\
f
\end{array}\right)= & \varepsilon\left(\begin{array}{c}
w_{2(m, n)}^{(1)} \\
f_{2(m, n)}^{(1)}
\end{array}\right) \sin m x \sin n y+\varepsilon^{2}\left(\begin{array}{c}
w_{1(m, 2 n)}^{(2)} \\
f_{1(m, 2 n)}^{(2)}
\end{array}\right) \cos n y \sin m x \\
& +\varepsilon^{3}\left[\left(\begin{array}{c}
w_{2(m, 3 n)}^{(3)} \\
f_{2(m, 3 n)}^{(3)}
\end{array}\right) \sin m x \sin 3 n y+\left(\begin{array}{c}
w_{2(m, n)}^{(3)} \\
f_{2(m, n)}^{(3)}
\end{array}\right) \sin m x \sin n y\right]+\cdots
\end{aligned}
$$

Equation (4.40) determines the displacement and the corresponding Airy stress functions.

\section{Values of Independent Variables at Maximum Displacement}

The analysis henceforth will be concerned with the displacement components that are partly in the shape of the imperfection or partly in the shape of $\sin m x \sin 3 n y$. In this respect, we neglect the displacements of order $\mathcal{O}\left(\varepsilon^{2}\right)$ in (4.40) so that the displacement becomes

$$
\begin{aligned}
w= & \varepsilon w_{2(m, n)}^{(1)} \sin m x \sin n y+\varepsilon^{3}\left\{w_{2(m, n)}^{(3)} \sin m x \sin n y\right. \\
& \left.+\Omega w_{2(m, 3 n)}^{(3)} \sin m x \sin 3 n y\right\}+\mathcal{O}\left(\varepsilon^{4}\right)
\end{aligned}
$$

where,

$$
\Omega=0 \text { or } 1
$$


When $\Omega=0$, we get the exact displacement that is purely in the shape of imperfection, but when $\Omega=1$, we get the resultant displacement incorporating the modes $\sin m x \sin 3 n y$ and $\sin m x \sin n y$.

Since the displacement $w$ in (5.1) depends on $x$ and $y$ then, the conditions for maximum displacement are as follows

$$
w_{, x}=w_{, y}=0
$$

We now let $x_{a}$ and $y_{a}$ be critical values of $x$ and $y$ respectively at maximum displacement.

From (5.1), using (5.2), we see that for maximum displacement,

$$
x_{a}=\frac{\pi}{2 m} ; \quad y_{a}=\frac{\pi}{2 n}
$$

where (5.3) are the least nontrivial values of $x_{a}$ and $y_{a}$.

\section{Maximum Displacement}

The maximum displacement is obtained from (5.1) at the critical values of $x$ and $y$ where $w$ has a maximum value. Hence, the value of $w$ at these values becomes

$$
w_{a}=\varepsilon w_{2(m, n)}^{(1)}(\lambda)+\varepsilon^{3}\left\{w_{2(m, n)}^{(3)}(\lambda)-\Omega w_{2(m, 3 n)}^{(3)}(\lambda)\right\}+\cdots
$$

Meanwhile, (6.1) can be recast as

$$
w_{a}=\varepsilon c_{1}+\varepsilon^{3} c_{3}+\mathcal{O}\left(\varepsilon^{4}\right)
$$

where,

$$
c_{1}=w_{2(m, n)}^{(1)}(\lambda), \quad c_{3}=w_{2(m, n)}^{(3)}(\lambda)-\Omega w_{2(m, 3 n)}^{(3)}(\lambda)
$$

\section{Static Buckling Load}

The static buckling load, $\lambda_{s}$ according to [24] [25] and [26], is obtained from the maximization

$$
\frac{\mathrm{d} \lambda}{\mathrm{d} w_{a}}=0
$$

The usual procedure (as in [22] [26] and [27]), is to first reverse the series (6.2) in the form

$$
\varepsilon=d_{1} w_{a}+d_{3} w_{a}^{3}+\cdots
$$

By substituting (6.1) into (7.2) and equating the coefficients of powers of orders of $\varepsilon$, we get

$$
d_{1}=\frac{1}{c_{1}}, \quad d_{3}=-\frac{c_{3}}{c_{1}^{4}}
$$

The maximization in (7.1) easily follows from (7.2) where $w_{a}$ is now being substituted for $w$ to yield, after some simplifications,

$$
\varepsilon=\frac{2}{3 \sqrt{3}} \sqrt{\frac{c_{1}}{c_{3}}}
$$

On substituting into (7.4), using (6.3) and simplifying, we get 


$$
\begin{aligned}
& \left\{\left(m^{2}+n^{2} \xi\right)^{2}+\left(\frac{m^{2} A}{m^{2}+n^{2} \xi}\right)^{2}-\lambda_{S}\left(\frac{1}{2} \alpha m^{2}+n^{2} \xi\right)\right\}^{\frac{3}{2}} \\
& =\frac{3 \sqrt{3}}{2} \lambda_{S}(\varepsilon \bar{a})\left(\frac{1}{2} \alpha m^{2}+n^{2} \xi\right) \Psi_{0}
\end{aligned}
$$

This determines the static buckling load $\lambda_{s}$ of the circular cylindrical shell structure, and the determination is implicit in the load parameter $\lambda_{s}$,

where,

$$
\begin{aligned}
& \Psi_{0}=\left(\Theta_{2} l_{01}-\Theta_{3} l_{0}\right) \sqrt{1-\frac{\Omega Q_{02}}{Q_{01}}}, Q_{01}=\frac{\left(\Theta_{2} l_{01}-\Theta_{3} l_{0}\right)}{\varphi_{0}^{2}} \\
& Q_{02}=\frac{\left(\Theta_{0} l_{0}-\Theta_{1} l_{01}\right)}{\varphi_{0}^{2}}, \varphi_{0}^{2}=\left[\left(m^{2}+n^{2} \xi\right)^{2}+\left(\frac{m^{2} A}{m^{2}+n^{2} \xi}\right)^{2}-\lambda_{S}\left(\frac{1}{2} \alpha m^{2}+n^{2} \xi\right)\right]
\end{aligned}
$$

\section{Results and Discussion}

The result (7.5) is asymptotic in nature. The results of the classical buckling load $\lambda_{C}$, and that of the cylindrical shell structure are as seen in (3.9), whereas, the corresponding displacement and Airy Stress function of the structure are as in (3.10). Similarly, the static buckling load $\lambda_{S}$, is as shown in (7.5). A computer program in MATLAB gives the relationship between the static buckling load $\lambda_{S}$, and the imperfection parameter $\varepsilon$, at $\Omega=0$ or $\Omega=1$, and where we have fixed the following as $\alpha=1, A=0.2, H=0.2, \bar{a}=0.02, \bar{\xi}=0.8, \quad m=1$ and $n=1$ is as shown in Table 1 .

A careful appraisal of the graph of Figure 1, shows that static buckling load $\lambda_{s}$, decreases with increased imperfection parameter $\varepsilon$. This is expected. In other words, static buckling load $\lambda_{\mathrm{s}}$ increases with less imperfection. The value of static buckling load $\lambda_{S}$ is higher when the buckling mode is a combination of the modes in the shape of imperfection ( $\sin m x \sin n y)$ and shape of other geometric form ( $\sin m x \sin 3 n y)$, i.e. $(\Omega=1)$ compared to the case when the buckling mode is in the shape of imperfection $(\sin m x \sin n y)($ i.e. $\Omega=0$ ).

Table 1. Relationship between static buckling load, $\lambda_{S}$ and imperfection parameter, $\varepsilon$ for some values of $\Omega$ of the circular cylindrical shell structure using Equation (7.5).

\begin{tabular}{ccc}
\hline$\varepsilon$ & \multicolumn{2}{c}{$\lambda_{s}$} \\
\hline 0.0100 & 2.4945 & $\Omega=1$ \\
0.0200 & 2.4944 & 2.4954 \\
0.0300 & 2.4943 & 2.4953 \\
0.0400 & 2.4942 & 2.4952 \\
0.0500 & 2.4941 & 2.4951 \\
0.0600 & 2.4940 & 2.4949 \\
0.0700 & 2.4939 & 2.4948 \\
0.0800 & 2.4937 & 2.4946 \\
0.0900 & 2.4936 & 2.4944 \\
0.1000 & 2.4933 & 2.4942 \\
\hline
\end{tabular}




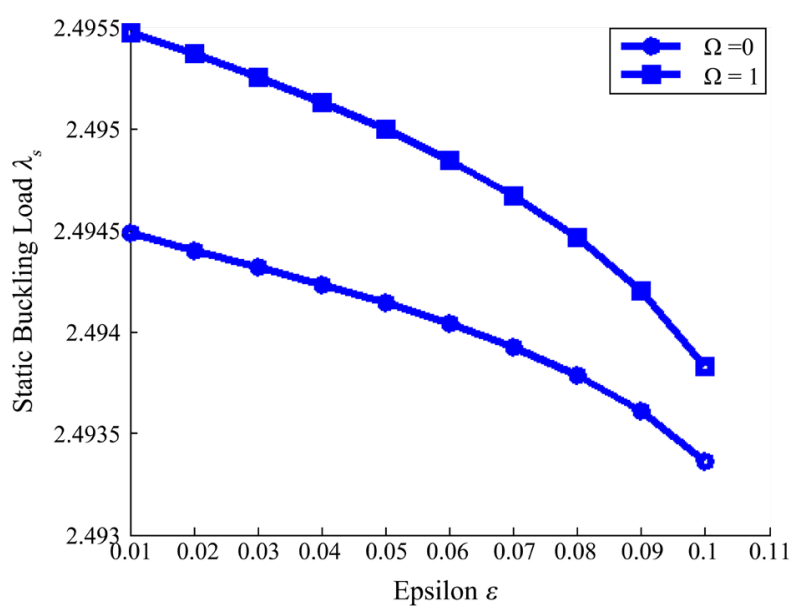

Figure 1. Graph of Static buckling load $\lambda_{S}$, as a function of imperfection parameter, $\varepsilon$ for some values of $\Omega$ of the circular cylindrical shell structure using Table 1 .

\section{Conclusion}

This analysis has analytically determined the maximum of the out-of-plane normal displacement of a finite imperfect cylindrical shell trapped by a static load. We have used the techniques of perturbation and asymptotics to derive an implicit formula for determining the static buckling load of the cylindrical shell investigated. The formulation contains a small parameter depicting the amplitude of the imperfection and on which all asymptotic series are expanded. Such an analytical approach can be duplicated for other structures including toroidal shell segments and plates.

\section{Conflicts of Interest}

The authors declare no conflicts of interest regarding the publication of this paper.

\section{Acknowledgements}

This paper is dedicated to the memory of late EZIKE OGBUEHI FELIX IKENNA OZOIGBO, who died on $20^{\text {th }}$ day of July, 2019 and to be buried on $22^{\text {nd }}$ day of November, 2019.

\section{References}

[1] Xu, X.S., Ma, J.Q., Li, C.W. and Chu, H.J. (2009) Dynamic Local and Global Buckling of Cylindrical Shells under Axial Impact. Engineering Structures, 31, 1132-1140. https://doi.org/10.1016/j.engstruct.2009.01.009

[2] Fan, H.G., Chen, Z.P., Feng, W., Fan, Z. and Cao, G.W. (2015) Dynamic Buckling of Cylindrical Shells with Arbitrary Axisymmetric Thickness Variation under Time Dependent External Pressure. International Journal of Structural Stability and Dynamics, 15, 21 p. https://doi.org/10.1142/S0219455414500539

[3] Koiter, W.T., Elishakoff, I.Y.W. and Li, Y.W. (1994) Buckling of an Axially Compressed Cylindrical Shell of Variable Thickness. International Journal of Solids Structures, 31, 797-805. https://doi.org/10.1016/0020-7683(94)90078-7 
[4] Hutchinson, J.W. and Amazigo, J.C. (1967) Imperfect-Sensitivity of Eccentricity Stiffened Cylindrical Shells. AIAA Journal, 5, 392-401.

https://doi.org/10.2514/3.3992

[5] Budiansky, B. and Roth, R.S. (1962) Axisymmetric Dynamic Buckling of Clamped Shallow Spherical Shells. Collected Papers on Stability of Shell Structures, NASA TN D-1510, Washington DC.

[6] Budiansky, B. and Hutchinson, J.W. (1970) Buckling Progress and Challenge in Process of Trends in Solid Mechanics. University Press Delft, Delft, 93-116.

[7] Roth, R. and Klosner, J. (1964) Nonlinear Response of Cylindrical Shells Subjected to Dynamic Axial Loads. AIAA Journal, 2, 1788-1794. https://doi.org/10.2514/3.2666

[8] Amazigo, J.C. and Frazer, W.B. (1971) Bucklng under External Pressure of Cylindrical Shells with Dimple-Shaped Initial Imperfection. International Journal of Solids Structures, 7, 883-900. https://doi.org/10.1016/0020-7683(71)90070-9

[9] Budiansky, B. and Amazigo, J.C. (1968) Initial Post-Buckling Behaviour of Cylindrical Shell under External Pressure. Journal of Mathematical Physics, 47, 233-235. https://doi.org/10.1002/sapm1968471223

[10] Ette, A.M. and Onwuchekwa, J.U. (2007) On the Static Buckling of an Externally Pressurized Finite Circular Cylindrical Shell. Journal of the Nigerian Association of Mathematical Physics, 11, 323-332. https://doi.org/10.4314/jonamp.v11i1.40226

[11] Lockhart, D. and Amazigo, J.C. (1975) Dynamic Buckling of Externally Pressurized Imperfect Cylindrical Shells. Journal of Applied Mechanics, 42, 316-320. https://doi.org/10.1115/1.3423574

[12] Bich, D.H., Dung, D.V., Nam, V.H. and Phuong, N.T. (2013) Nonlinear Static and Dynamic Buckling Analysis of Imperfect Eccentrically Stiffened Functionally Graded Circular Cylindrical Thin Shells under Axial Compression. International Journal of Mechanical Science, 74, 190-200. https://doi.org/10.1016/j.ijmecsci.2013.06.002

[13] Paimushin, V.N. (2016) Static and Dynamic Buckling Modes of Spherical Shells Subjected to External Pressure. Russian Mathematics, 60, 37-46. https://doi.org/10.3103/S1066369X1604006X

[14] Paimushin, V.N. (2017) Static and Dynamic Beam Buckling Shapes of Long Orthotropic Cylindrical Shells Undergoing External Pressure. Journal of Applied Mathematics and Mechanics, 72, 1014-1027.

[15] Lukankin, S.A. and Paimushin, V.N. (2014) Static and Dynamic Beam Buckling Shapes of Long Orthotropic Cylindrical Shells under External Pressure. Izv. RAS. MTT, No. 1, 108-128.

[16] HuyBich, D., Dung, D.V., Namb, V.H. and Phuong, N.T. (2013) Nonlinear Static and Dynamic Buckling Analysis of Imperfect Eccentrically Stiffened Functionally Graded Circular Cylindrical Thin Shells under Axial Compression. International Journal of Mechanical Sciences, 74, 190-200. https://doi.org/10.1016/j.ijmecsci.2013.06.002

[17] Ette, A.M. (2003) Buckling of a Cylindrical Shell Pressurized by an Impulse. Journal of the Nigerian Mathematical Society, 22, 82-110.

[18] Ette, A.M. (2004) On the Dynamic Buckling of Stochastically Imperfect Finite Cylindrical Shells under Step Loading. Journal of the Nigerian Association of Mathematical Physics, 8, 35-46. https://doi.org/10.4314/jonamp.v8i1.39971

[19] Ette, A.M. (2006) On the Dynamic Buckling of Lightly Damped Cylindrical Shell Modulated by a Periodic Load. Journal of the Nigerian Association of Mathematical Physics, 10, 327-344. https://doi.org/10.4314/jonamp.v10i1.40141 
[20] Ette, A.M. (2008) Perturbation Technique on the Dynamic Buckling of a Lightly Damped Cylindrical Shell Axially Stressed by an Impulse. Journal of the Nigerian Association of Mathematical Physics, 12, 103-120. https://doi.org/10.4314/jonamp.v12i1.45493

[21] Ette, A.M. (2008) Initial Post-Buckling of Toroidial Shell Segments Pressurized by External Load. Journal of the Nigerian Association of Mathematical Physics, 12, 121-132. https://doi.org/10.4314/jonamp.v12i1.45494

[22] Amazigo, J.C. (1974) Asymptotic Analysis of the Buckling of Externally Pressurized Cylinders with Random Imperfections. Quarterly of Applied Mathematics, 32, 429-442. https://doi.org/10.1090/qam/99693

[23] Batdorf, S.B. (1947) A Simplified Method of Elastic Stability Analysis for Thin Cylindrical Shells. NACA Report, 874.

[24] Budiansky, B. (1966) Dynamic Buckling of Elastic Structures, Criteria and Estimation. In: Dynamic Stabilities of Structures, Hermann Pergamon, Oxford, 83-106.

[25] Hutchinson, J.W. and Budiansky, B. (1966) Dynamic Buckling Estimates. AIAA Journal, 4, 525-530. https://doi.org/10.2514/3.3468

[26] Amazigo, J.C. and Ette, A.M. (1987) On a Two-Small Parameter Differential Equation with Application to Dynamic Buckling. Journal of Nigerian Mathematical Society, 6, 90-102.

[27] Amazigo, J.C. (1971) Buckling of Stochastically Imperfect Columns on Nonlinear Elastic Foundation. Quarterly of Applied Mathematics, 29, 403-409. https://doi.org/10.1090/qam/99755 